\title{
La Responsabilité Sociale de l'Entreprise comme « représentation » de l'économie et du droit Franck Cochoy
}

\section{Titre anglais : Corporate Social Responsibility: representing the economy and the law}

Résumé : Les pratiques de Responsabilité Sociale renouvellent la «représentation» de l'économie et du droit: d'une part, ces pratiques considèrent les règles (publiques et privées) non comme des obstacles, mais comme autant de ressources avec lesquelles il est possible de jouer; d'autre part, ces pratiques nous montrent qu'en l'absence de règles publiques, les acteurs sont capables d'inventer eux-mêmes les règles dont ils ont besoin. Enfin, les autorités publiques, loin d'être en reste, essayent de tourner le jeu des entreprises à leur avantage, dans l'espoir de définir les contours d'une représentation de l'économie où les règles du privé pourraient servir de bases à de futures régulations publiques. (704 signes).

Mots clés : responsabilité sociale de l'entreprise, capture, certification, contrefaçon, démocratie technique, normalisation, service public

Abstract: Corporate Social Responsibility Practices renew the representation of the economy and the law: on the one hand, such practices take rules (be they public or private) not as obstacles but rather as strategic resources in the economic game; on the other hand, these practices show that when public rules are lacking, private actors are capable to invent the rules they need. Finally, public authorities are not inactive. Rather, they attempt to take advantage of the business game, in order to set up a representation of the economy where private rules may serve as the foundation of new public regulation.

Key words: Corporate Social Responsibility, capture, certification, fake, public service, standardization, technical democracy.

Biographie. Franck Cochoy, 42 ans, Professeur de sociologie à l'Université Toulouse II et membre du CERTOP (UMR CNRS 5044), consacre ses travaux à l'étude des médiations marchandes (marketing, packaging, distribution, normalisation...). Il est l'auteur de Une histoire du marketing, Paris, La Découverte, 1999 et de Une sociologie du packaging ou l'âne de Buridan face au marché, Paris, PUF, 2002.

\section{Coordonnées :}

\section{Bureau :}

\section{CERTOP}

Maison de la recherche,

5, allées Antonio Machado,

31058, Toulouse Cedex.

Tél. 0561503977

Fax. 0561504963

\section{Domicile :}

8, Rue de l'Aurore

31500 Toulouse

Tél : 0562160609

Email : cochoy@univ-tlse2.fr 
L'approche de la pertinence des théories, des formats et des règles de l'économie a été profondément renouvelée par la conception de l'économie comme «science performative». Selon cette conception, plutôt que d'opposer sans cesse l'économie théorique (economics) à l'économie réelle (economy), l'idéologie du marché aux échanges effectifs, la fiction des règles à la réalité des pratiques, mieux vaut s'intéresser à la façon dont les unes sont « performées » par les autres ${ }^{1}$. S'inspirant de la linguistique d'Austin, qui définit un énoncé performatif comme un énoncé capable de dire et de faire ce qu'il dit simultanément, cette perspective de recherche propose de tracer les liens qui se nouent entre les conceptions et les pratiques de l'économie, par exemple en étudiant les textes et/ou les dispositifs (lois, logiciels, systèmes comptables...) qui permettent à ces conceptions de passer dans les faits. Une telle posture semble rejoindre l'effort symétrique qui, en postulant que les normes d'entreprise véhiculent un certain nombre de «représentations » plus ou moins explicites, suggère de remonter en sens inverse de la production normative concrète aux contenus qui leur donnent forme et sens - pour le dire en d'autres termes, si Austin montre que dire, c'est faire, on peut alors s'intéresser aussi à ce que faire veut dire ou ce que faire peut (vouloir) dire.

Nous proposons de compléter ces deux démarches convergentes et complémentaires en nous situant à mi-chemin, pour explorer un autre sens des deux idées de «performation » et de « représentation» qui leur servent de fondations respectives. «Performer », c'est à la fois « réussir» une action et «jouer» un script; assurer une «représentation» - ou une « performance », en anglais - c'est à la fois incarner un message et accomplir une action. Ainsi, dans la suite de notre exposé, nous nous intéresserons à la performation/représentation de l'économie et des règles dans le cadre des politiques de "Responsabilité Sociale des Entreprises » (RSE). La RSE consiste, pour une firme, à se doter de standards (en matière d'environnement, de santé et de sécurité et de conditions de travail) censés conduire l'entreprise en question à agir «au-delà de ses obligations légales $»^{2}$. Nous voudrions montrer que la représentation/performance de la RSE engage non seulement leur contenu, mais aussi ces règles elles-mêmes - le rôle qu'on leur donne et le personnage qu'on leur fait jouer. Le jeu sur les mots, ici, loin d'être une coquetterie de sociologue, est consubstantiel à la pratique des acteurs : la RSE de l'entreprise est bien une affaire de "performance », au double sens de jeu de rôle (Cf. l' «affichage »d'une orientation éthique) et/ou d'enjeu économique (Cf. l'« efficacité » économique de l'action implicitement visée).

La vulgate ultra-libérale postule que les règles font obstacle au fonctionnement du marché, ce qui amène ses partisans les plus zélés (mais aussi les plus naïfs) à demander toujours plus de déréglementation. Pourtant, les pratiques de RSE donnent à voir une tout autre conception des règles de la part d'acteurs pourtant engagés au cœur de l'économie libérale : d'une part, les entreprises qui promeuvent la RSE considèrent les règles (publiques et privées) non comme des obstacles, mais comme autant de ressources avec lesquelles il est possible de jouer; d'autre part, en l'absence de règles publiques, ces entreprises nous montrent que les acteurs sont capables d'inventer eux-mêmes celles dont ils ont besoin. Par ailleurs, dans la vaste représentation d'une économie socialement responsable, les autorités publiques ne sont pas en reste : nous verrons que si les entreprises jouent avec le droit, les instances politiques essayent

1. Cf. M. CALLON (ed.), The Laws of the Markets, Oxford, Blackwell, 1998.

2. Cf. Jacques IGALENS \& Michel JORAS, \& La responsabilité sociale de l'entreprise, Paris, Les Éditions d'Organisation, 2002 ; David VOGEL, The Market for Virtue. The Potential and Limits of Corporate Social Responsibility, Brookings Institution Press, 2005. 
de tourner ce premier jeu à leur avantage, dans l'espoir de définir les contours d'une représentation de l'économie où les règles du privé pourraient servir de bases à de futures régulations publiques ${ }^{3}$.

\section{Représenter le social dans l'économie libérale}

La promotion des politiques de RSE intervient au confluent de toute une série de mouvements. En 1987 est paru le rapport Brundtland. Ce rapport, réalisé pour la Commission des Nations Unies sur l'Environnement et le Développement, a lancé l'idée de développement durable et de prise en compte de l'impact environnemental des activités économiques, bientôt étendu à leur impact social. En 1996 a été élaboré le standard SA 8000, qui propose de certifier par audit tierce partie le respect d'un certain nombre d'engagements volontaires des entreprises en matière de pratiques sociales. En 1997 a été lancée la Global Reporting Initiative, qui promeut un ensemble de principes comptables destinés à fixer les règles nécessaires au « reporting sociétal » des entreprises à destination de leurs diverses parties-prenantes. En 2000 les Nations Unies ont mis au point le programme Global Compact qui offre le label des Nations Unies aux entreprises qui s'engagent à respecter un certain nombre de principes sociaux, tandis que l'OCDE a révisé la même année ses principes directeurs pour faire une place aux questions de RSE. En 2001 la France a adopté une loi sur Les nouvelles régulations économiques dont l'article 116 oblige les entreprises à compléter les informations économiques et financières de leur rapport annuel par d'autres informations de nature sociale et environnementale. En juillet 2001, la commission Européenne a publié un Livre Vert pour «Promouvoir un cadre européen pour la responsabilité sociale des entreprises » et consulter les acteurs économiques sur ce thème. Plus récemment, en 2004, l'ISO a organisé une conférence sur la RSE et lancé un programme de travail sur ce thème avec pour horizon l'établissement d'une normalisation internationale.

Dans cette liste d'initiatives, il est bien difficile de démêler les contributions respectives des acteurs privés et des instances publiques. Certes, on reconnaît derrière elles d'une part des instances politiques nationales (France), régionales (Europe) et internationales (Nations Unies, OCDE), et d'autre part des acteurs de la société civile comme des groupements d'ONG, de syndicats et de professionnels... sans oublier toutes les entreprises qui, tout au long de la période, ont adopté à titre individuel des codes de conduite volontaire et/ou se sont engagées dans divers programmes de RSE. Mais un examen plus serré révèle une réalité plus complexe : d'un côté, le Global compact et le Livre Vert européen en appellent explicitement à la contribution active des entreprises, d'un autre côté les groupements à l'origine des standards SA 8000 et GRI ont inclus dès leur origine (respectivement) des règles publiques (standards de l'OIT) et des partenaires publics (Programme des Nations Unies pour l'Environnement).

Ce mélange des acteurs à l'origine des diverses initiatives est le symptôme du même problème que tous tentent de relever : trouver des règles efficaces dans un univers qui en manque, dans un contexte de mondialisation où chacun sait qu'aucun type d'instance ne peut, à elle seule, construire et surtout mettre en œuvre ces règles qui font défaut à tous. Aujourd'hui, les institutions publiques (États, Europe...) ne disposent ni de la légitimité ni des moyens nécessaires pour définir et imposer des régulations a priori et englobantes. Certains acteurs

3. Cet article s'inscrit dans le cadre d'une recherche financée par la Commission Européenne, Ème PCRD, Priorité 7, Citizens and governance in a knowledge based society, projet ESTER, juin 2004-juin 2007, Isabelle DAUGAREILH, dir. 
privés profitent de ce vide pour devancer le droit, ou pour le jouer autrement, en free lance, tandis qu'entre les deux des organismes privés ou des institutions internationales tentent de susciter ou de canaliser ces initiatives pour «réglementer par le bas » une économie que personne ne parvient plus à réguler par le haut. En l'absence de gouvernement mondial, chaque opérateur (privé ou national) fonctionne comme une «entreprise » de régulation ; en retour, la juxtaposition des initiatives contribue autant à l'émergence d'un nouvel ordre commun qu'à l'instauration d'une concurrence par les règles.

\section{Rejouer le service public, jouer aux autorités sanitaires}

La RSE permet aux entreprises de «re-présenter » des règles publiques, au sens littéral du terme, comme le montre l'exemple d'un ancien monopole public de l'électricité dont le capital et le marché ont récemment été ouverts. Cette entreprise trouve dans la RSE un moyen de proposer «à nouveau» mais «ailleurs » et «pour d'autres raisons » les règles du service public qui fondaient jusque là son activité, et que vient menacer l'apparition de la concurrence et l'ouverture récente du capital de l'entreprise au secteur privé. L'accord relatif à la RSE signé dans ce groupe avec les partenaires sociaux précise ainsi :

«Les signataires considèrent que l'accès à l'électricité est un facteur majeur de développement économique et social, et un facteur clé de lutte contre la pauvreté [...] Le Groupe X et les sociétés qui le composent prennent des initiatives, ou s'y associent en partenariat, pour favoriser dans différents pays, et en particulier dans les régions où elles sont implantées, un meilleur accès des populations à l'électricité » (Extrait de l'accord relatif à la RSE de ce groupe).

On retrouve dans cet accord la notion fondamentale d'égalité d'accès et d'universalité des prestations chère à la doctrine du service public. Mais ici, l'ancienne «obligation de service public », qui ne s'impose en rien à l'étranger, devient un engagement volontaire, dont l'entreprise entend tirer parti à la fois pour conforter son identité, souder ses salariés, et conquérir des marchés au nom d'engagements susceptibles d'être valorisés comme un avantage concurrentiel spécifique.

Le second cas de figure est porté par l'entreprise Philip Morris, dont le «code marketing » illustre de façon exemplaire ce qu'est une démarche de RSE... mais aussi les ambiguïtés dont ce type de démarche est (inévitablement ?) porteur. Dans ce code de marketing, le fabricant de tabac proclame très explicitement :

\footnotetext{
«Les avertissements sanitaires sont un élément obligatoire de l'emballage et de la publicité de tous nos produits, qu'il existe ou non une législation nationale en matière d'avertissements sanitaires. Toute publicité doit respecter les dispositions des lois applicables relatives aux avertissements, aux informations concernant les taux de goudrons et de nicotine, et toute autre mention devant apparaître sur les publicités de produit du tabac. [...] Lorsque la loi ne spécifie pas le texte, la taille, le format ou l'emplacement des avertissements dans la publicité, PMI place volontairement des avertissements clairs et très visibles dans ses publicités. »
}

Autrement dit et conformément à la philosophie des démarches de RSE, le groupe Philip Morris fait preuve d'une indiscutable responsabilité sociale, d'une part en se montrant conscient et soucieux des problèmes de santé que pose le produit qu'il commercialise, d'autre part en se montrant respectueux du droit et surtout en prenant des engagements qui vont «audelà » de ses obligations légales pour assumer ses responsabilités «sociétales ». Pourtant, la contradiction manifeste entre cette orientation et l'intérêt économique de l'entreprise porte à s'interroger. L'un des points d'application les plus «marquants » de cette politique concerne l'engagement de Philip Morris à lutter contre le tabagisme de la jeunesse. Depuis 1999, le groupe appose sur ses paquets un message volontaire «les mineurs ne doivent pas fumer». 
Faut-il s'en réjouir, jusqu'à rêver que la RSE puisse amener certaines activités économiques discutables à s'autodétruire ? Rien n'est moins sûr. D'une part, l'apposition d'un message volontaire difficilement discernable des autres messages sanitaires proposés par le droit mais étonnamment proche de leur esprit et de leur rédaction ${ }^{4}$ permet de se demander si nous ne sommes pas en présence d'une contrefaçon du droit dont on chercherait à capter l'autorité et la légitimité ; d'autre part, on peut s'interroger sur les intentions d'une telle captation: la restriction du tabac à un public adulte ne contribue-t-elle pas à encourager les jeunes à défier les autorités publiques et à adopter un comportement contraire aux mises en garde sanitaires, tant l'on sait que les mineurs aspirent justement à cesser de l'être ? À tout le moins, la RSE appliquée au marché du tabac apparait clairement comme un moyen pour les entreprises concernées d'anticiper voire de prévenir des mises en cause juridiques et des régulations plus contraignantes ${ }^{5}$.

\section{Représenter le droit et la dimension sociale de l'économie : certification et reporting sociétal}

Si les entreprises prises isolément s'engagent en matière de RSE, certains acteurs investissent cette dernière comme un véritable marché en proposant aux entreprises soucieuses de responsabilité sociale un certain nombre de principes et de services «clé en main». Le référentiel «SA $8000 »$ relève de cette catégorie ${ }^{6}$. Ce dispositif propose à la fois un certain nombre d'orientations socialement responsables et la certification par audit tierce partie des entreprises qui s'engagent à s'y conformer. Officiellement présenté le 15 octobre 1997, ce dispositif a été mis au point sous l'égide du Council on Economic Priorities, une ONG qui œuvre depuis 1969 pour l'amélioration du comportement social et environnemental des entreprises. Le CEP a confié l'élaboration du référentiel à un organisme créé pour l'occasion, le CEPAA (Council on Economic Priorities Accreditation Agency), qui rassemble des ONG, des syndicats, des entreprises et des universitaires, sur un modèle qui n'est pas sans rappeler le fonctionnement des organismes officiels de normalisation. Le standard SA 8000 entend promouvoir une responsabilité sociale des entreprises «au-delà » des obligations légales locales en vigueur dans les pays où elles opèrent en s'appuyant notamment sur les principes généraux de l'OIT'.

Mais là où l'OIT se contente d'édicter un certain nombre de points difficilement applicables et vérifiables, car relevant de la seule responsabilité des États, le référentiel SA 8000 tente de faire mieux, en situant l'effort au niveau plus local, et donc plus facilement contrôlable, des organisations productives. L'effort est double : d'une part le référentiel exige la mise en place

4. Cf. par exemple les mentions «Protégez les enfants : ne leur faîtes pas respirer votre fumée » et «Fumer pendant la grossesse nuit à la santé de votre enfant » proposées par la directive 2001/37/CE du 5 juin 2001.

5. Pour une analyse détaillée de ce cas Cf. Franck COCHOY, Loïc Le DANIEL, \& et Jacques CRAVE, «Le grand chevron rouge et les 282 petits chevreaux, ou l'emballage des cigarettes comme dispositif de captation », Terrains et travaux (à paraître, 2006).

6. On pourrait citer aussi les agences de rating. Sur ce point, Cf. Jean-Pascal GOND, \& Bernard LECA, «La construction de la notation sociale des entreprises, ou l'histoire d'ARESE », Sciences de la société, 62, mai 2004, p. 189-207 ; Stéphanie GIAMPORCARO, «L'investissement socialement responsable en France : un outil au service d'une action politique par la consommation ? », Sciences de la société, 62, mai 2004, p. 169-187.

7. Plus précisément, le référentiel se réfère non seulement aux conventions de l'OIT, mais aussi à la Déclaration Universelle des Droits de l'Homme et à la Convention des Nations Unies sur les droits de l'Enfant. 
de dispositifs organisationnels propres à "gérer» sa mise en œuvre (nomination d'un représentant de la direction chargé du respect de la norme, mise en place d'une traçabilité des pratiques sociales de l'entreprise...) ; d'autre part et surtout, le référentiel incorpore le principe de l'audit tierce partie assuré par des sociétés d'audit accréditées par le CEPAA. Le résultat d'une telle combinaison de principes juridiques et de techniques managériales est ambigu. D'un côté, le référentiel SA 8000 semble apte à promouvoir les exigences du droit international plus efficacement que ce droit lui-même, en connectant les questions de droit à des leviers marchands et managériaux. D'un autre côté, la manière dont ces règles officielles sont promues pose un double problème : d'une part, le nom même du dispositif, SA 8000, en sonnant comme ISO 9000 (au risque de la contrefaçon), pourrait faire croire que l'on est en présence d'un référentiel établi par un organisme officiel de normalisation et selon les mêmes procédures, alors qu'il s'agit d'un standard privé dont la composition et le fonctionnement sont à la discrétion des organismes impliqués ; d'autre part, en certifiant la conformité des pratiques des entreprises à des principes largement empruntés à l'OIT, le référentiel SA 8000 vend le droit international sous des couleurs privées, au risque de présenter comme un privilège et comme un avantage compétitif les exigences de conventions qui s'imposent aux entreprises des pays qui les ont ratifiées.

Toutefois, le dispositif SA 8000 possède un grand mérite : en mettant au point un «corps d'inspection » privé censé contrôler le respect sinon du droit international du travail, du moins de standards qui s'en inspirent, il pointe la carence d'un dispositif public analogue du côté des autorités publiques. Ce qui vaut du côté du contrôle se retrouve du côté des règles ellesmêmes : le développement de la RSE témoigne d'un véritable «besoin de droit » des acteurs privés engagés dans la compétition mondiale. L'urgence d'un tel besoin semble telle que les acteurs préfèrent «légiférer » par eux-mêmes plutôt que d'attendre l'improbable venue d'une réglementation et d'une police publiques au niveau global. Ainsi, tandis que le standard SA 8000 a esquissé la mise au point d'un système d'inspection, la Global Reporting Initiative (GRI) s'est attachée à définir des règles de comptabilisation standard (ou «reporting») des actions socialement responsables, de façon à assurer la crédibilité et la comparabilité des informations disponibles, sans lesquelles tous les rapports portant sur des actions socialement responsables risquent d'apparaître comme autant d'opérations de communication vagues, incommensurables entre elles, et difficilement contrôlables.

La GRI ne peut se comprendre sans référence aux marchés financiers. D'une part, c'est bien l'émergence des fonds d'investissement éthiques et d'un «rating sociétal » des entreprises à destination des investisseurs qui fonde le développement de «rapports sociétaux » censés servir de «vitrine sociale » aux entreprises, à côté des classiques rapports financiers faisant office de «tableau d'honneur économique ${ }^{8}$. D'autre part et comme l'ont bien noté Michel Capron et Françoise Quairel ${ }^{9}$, la mise au point de la GRI comme standard de comptabilité sociétale d'origine privée s'inspire directement d'une initiative similaire prise en matière de comptabilité des produits financiers : tout se passe comme si les promoteurs de la GRI avaient cherché à « rejouer » une scène à succès dans l'espoir de recueillir le même accueil. Dès 1973, des professionnels de la comptabilité et de l'audit de plusieurs pays avaient fondé à Londres un

8. Cf. Frédéric LORDON, Et la vertu sauvera le monde... Après la débâcle financière, le salut par l' "éthique”? Paris, Raisons d'agir, 2003.

9. Cf. Michel CAPRON, \& Françoise QUAIREL, «Reporting sociétal : limites et enjeux de la proposition de normalisation internationale "global reporting initiative" ", Congrès de l'Association Francophone de Comptabilité, Louvain la Neuve, 22 mai, 2003. 
International Accounting Standard Committee (IASC) afin de promouvoir la convergence des pratiques comptables des pays de l'OCDE, de construire des standards susceptibles d'être acceptés internationalement, et de surmonter les difficultés que posaient l'existence de comptabilités multiples tant aux entreprises transnationales qu'aux grands cabinets d'audit. Cette initiative s'est traduite par la mise au point, à partir de 1994, de normes qui proposent d'évaluer les actifs financiers à leur «fair value » ou valeur liquidative (c'est-à-dire « combien cela vaudrait si on le vendait») par opposition à la comptabilisation au coût historique qui prévalait jusqu'alors ( « combien cela a coûté de l'acquérir ») ${ }^{10}$. De façon similaire, la GRI a été lancée en 1997 par le CERES (Coalition for Environmentally Responsible Economies), un organisme regroupant des ONG centrées sur les questions d'environnement, des investisseurs institutionnels et autres gestionnaires de fonds éthiques, des organisations syndicales et religieuses, afin d'établir et de diffuser des standards en matière de publication de rapports environnementaux et sociaux. Cette initiative, financée par des fondations américaines, a rassemblé des professionnels de la comptabilité, de l'audit et des milieux d'affaires mais aussi de l'action syndicale qui ont cherché, tout comme l'IASC, à surmonter la carence de règles internationales en proposant son propre système comptable comme standard susceptible de s'imposer à l'ensemble des autres acteurs.

On pourrait considérer l'IASC ${ }^{11}$ et la GRI comme des «gouvernements privés », dans la mesure où il s'agit d'institutions qui se donnent des représentations et des buts politiques, censés pallier la carence ou le retard des autorités publiques en matière de gestion des questions de démocratie technique ${ }^{12}$ à l'échelle internationale ${ }^{13}$. Les travaux de tels organismes dépassent le seul intérêt des acteurs privés, puisque les standards qu'ils proposent sont susceptibles de satisfaire les attentes de multiples parties prenantes, y compris publiques. Mais ces standards, en relevant de l'initiative privée, permettent aussi de relayer des préoccupations particulières : les normes de l'IASC relèguent la comptabilité comme outil de contrôle public ou comme support des obligations fiscales au second plan derrière une comptabilité prioritairement tournée vers l'information des actionnaires ou des investisseurs réels ou potentiels ; de même - et malgré des orientations très différentes - la GRI privilégie le point de vue des «parties prenantes » destinataires des rapports sociétaux (leur souci d'accès à de tels comptes-rendus) au détriment de la rigueur purement technique du système de comptabilité (l'exigence de nomenclatures précises et fiables).

10. Cf. Ève CHIAPELLO, «Les normes comptables comme institution du capitalisme. Une analyse du passage aux normes IFRS en Europe à partir de 2005 », Sociologie du Travail, 47, 3, juillet-septembre, p. 362-382, 2005 ; David MARTIN, «Un travail de mondialisation. L'écriture de la "juste valeur" comptable des entreprises », in Gilbert de TERSSAC et Daniel FILÂTRE (dir.), Les dynamiques intermédiaires au cour de l'action publique, Toulouse, Octarès, p. 225-234, 2005.

\section{L'IASC est devenue IASB en 2001.}

12. Par démocratie technique, on désigne l'extension des procédures de délibération politiques à la prise en compte des entités non humaines (sur ce point, Cf. Bruno LATOUR, Politiques de la nature, Paris, La Découverte, 1999).

13. Ce type de mouvement est ancien, comme le montre l'exemple des sociétés savantes engagées dans la standardisation du temps magnifiquement étudié par Zerubavel (Eviatar ZERUBAVEL, "The Standardization of Time: A Sociohistorical Perspective," American Journal of Sociology, 88, 1, 1982, p. 1-23) ; il est aussi d'une actualité brûlante, comme l'illustre le succès d'une entreprise comme Google, dont l'activité s'apparente à l'avènement d'un véritable «service public privé » à l'échelle globale (Nicole PÉNICAUT, «Vertige de l'information illimitée : et si le succès de Google tournait la tête de ses salariés et "usagers"? », Libération, jeudi 19 août 2004). 


\section{Vers un retour de la représentation politique ?}

Parce qu'il consiste à édicter des règles à usage public dont la définition, loin d'être soumise à un large débat, relève plutôt de l'initiative de forums privés, ce type de régulation confronte les autorités publiques à un véritable dilemme. D’un côté, ces autorités savent que les standards privés peuvent servir de levier à des intérêts particuliers plutôt qu'à la promotion de l'intérêt général, à l'écart d'un véritable contrôle démocratique. D'un autre côté, les mêmes autorités sont conscientes qu'elles auraient du mal à proposer des règles plus pertinentes, en raison de leur propre éloignement vis-à-vis des acteurs impliqués et de leur manque d'expertise technique quant aux questions abordées. Or, si l'on en juge par le destin des normes de l'IASC, il semble que l'Union Européenne ait expérimenté un moyen de surmonter ce dilemme. En 2000, la Commission Européenne a décidé de confier à l'IASC l'élaboration de normes comptables destinées à fournir un cadre plus général que les normes américaines, tout en adoptant les principes de ces dernières, comme la «fair value ». Ainsi, «l'Europe est [...] passée directement d'un modèle traditionnel de décision nationale à une délégation de souveraineté au bénéfice d'un organisme privé à vocation mondiale ${ }^{14}$. Conformément à cette orientation, l'Europe a ensuite imposé ces normes comme référence pour toutes les entreprises européennes à compter de l'année 2005. Or, comme tout à l'heure, l'expérience acquise du côté de la sphère financière semble trouver son écho du côté de la RSE. Après avoir observé les différentes initiatives des entreprises et autres organismes internationaux en matière de RSE, la Commission Européenne a d'abord publié en 2001 un Livre Vert destiné à lancer une vaste consultation des parties intéressées puis, au vu des réponses reçues, organisé un forum qui s'est tenu en 2004, pour enfin adopter le 27 octobre 2004 une proposition de directive exigeant la publication annuelle, par les entreprises domiciliées en Europe, d'un « bilan de leur gouvernance d'entreprise dans leur rapport annuel $»^{15}$.

Comment donner sens aux initiatives que nous venons d'évoquer? George Stigler $(1971)^{16}$ avait souligné la capacité des entreprises à «capturer», grâce au lobbying, la régulation publique à leur profit. La RSE semble enrichir ce modèle, en ajoutant à la capture synchrone des lobbyistes une capture ex ante qui consiste pour les acteurs privés à tenter de prévenir, anticiper, orienter ou même préparer de futures régulations publiques, et même une capture $e x$ post, qui consiste pour les mêmes acteurs à convertir les règles auxquelles ils sont soumis en un lieu en autant d'engagements volontaires en un autre point de la planète où ces règles, nullement requises par le droit local, apparaissent alors comme des outils au service de la stratégie discrétionnaire de la firme ${ }^{17}$. Enfin et paradoxalement, les autorités publiques ne sont

14. Cf. Nicolas VÉRON, « Normalisation comptable internationale: une gouvernance en devenir », in Jacques MISTRAL, Christian de BOISSIEU, \& Jean-Hervé LORENZI, Les normes comptables et le monde post-Enron, Rapport du Conseil d'Analyse Économique, Paris, La documentation française, 2003, p. 123.

15. Pour une analyse détaillée de l'évolution de la stratégie européenne en matière de RSE, Cf. Franck COCHOY, Aurélie LACHÈZE, "Capture and counter-capture in Corporate Social Responsibility policies: Evidence from four transnational companies and the European Union," Market Making and Market Shaping in the Global Political Economy, Conference of the Political Economy Section of the German Political Science Association, Fern Universität, Hagen, Germany, 16-17 December 2005.

16. Cf. George J. STIGLER, "The theory of economic regulation," The Bell Journal of Economics and Management Science, 2, 1 (Spring), 1971, p. 3-21.

17. Cf. Isabelle DAUGAREILH \& Emmanuel POIRIER, «Les sources juridiques des règles appliquées aux travailleurs des entreprises transnationales à structure complexe », Syndicalisme et société, vol. 3, $\mathrm{n}^{\circ} 2,2000$, p. 83-111. L'exploitation des différentiels existant entre les différentes régulations nationales à des fins de 
pas en reste, mais semblent au contraire s'appuyer sur les initiatives privées censées les dépasser pour s'engager dans une véritable stratégie de «contre-capture ». Les deux exemples que nous avons évoqués montrent que cette stratégie articule deux moments. Dans un premier temps, les autorités publiques prennent paradoxalement le parti du «laisser-faire » : elles observent les pratiques existantes, encouragent les initiatives des acteurs, les invitent à développer ces standards (ou «soft laws ») susceptibles à la fois de porter des valeurs publiques (telle la responsabilité sociale), et de mieux correspondre aux situations visées que des règles générales construites à partir d'une expertise déficiente et à l'écart du terrain. Dans un deuxième temps, les autorités publiques inaugurent une nouvelle pratique de "faire laisserfaire » qui s'apparente à une véritable «contre-capture»: elles tentent de s'approprier ce mouvement, en l'organisant, puis en imposant à l'ensemble des acteurs les normes que certains d'entre eux ont contribué à construire comme nouvelles règles publiques ${ }^{18}$ (i.e. codification publique d'une norme privée).

Notons toutefois qu'il s'agit d'une stratégie fragile et risquée : le jeu «multipartite» sur le droit autour de la RSE s'apparente à une représentation incertaine, dont ni le script ni le dénouement ne sont donnés d'avance. Si l'Europe est parvenue à fonder une nouvelle régulation publique sur le travail de l'IASC en matière de produits financiers, elle semble hésiter à s'engager dans une voie analogue en matière de RSE, préférant temporiser, dans un contexte où les entreprises européennes semblent préférer se tourner plutôt vers des standards d'une plus vaste extension, comme le Global compact des Nations Unies ou les principes directeurs de l'OCDE. De façon symptomatique, dans une communication du 22 mars 2006 intitulée «Faire de l'Europe un pôle d'excellence en matière de Responsabilité Sociale des Entreprises », la Commission Européenne se retranche à nouveau derrière une RSE à l'initiative des acteurs privés qu'elle entend simplement animer et encourager autour d'un vague projet «d'alliance européenne pour la RSE »... dont tout projet de directive semble avoir disparu. Au total, la RSE comme représentation du droit et de l'économie se présente comme un étrange jeu de rôles et de masques, où les acteurs privés et publics se miment les uns les autres, échangent leurs positions et leurs discours, leurs outils et leurs objectifs, sans qu'il soit possible, à ce stade, de savoir s'il s'agit d'un jeu de dupes ou d'une expérimentation collective porteuse à terme d'un nouvel ordre juridique et économique mondial — d'un nouveau type de représentation, au sens politique du terme.

stratégie de RSE n'est pas sans rappeler la figure de l'arbitrage qui, sur les marchés financiers, consiste à obtenir des profits certains et sans risques en tirant parti des écarts de cours constatés pour un même actif financier d'un marché à l'autre.

18. Cette stratégie est explicitement évoquée et reprise dans les recommandations du rapport Delmas-Marsalet relatif à la commercialisation des produits financiers (Jacques DELMAS-MARSALET, Rapport relatif à la commercialisation des produits financiers, Paris, Ministère de l'Économie et des Finances, 2005). 